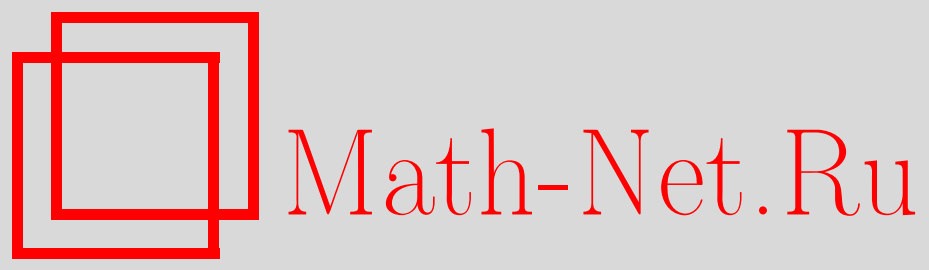

В. Ф. Молчанов, Разделение серий для гиперболоидов, Функи. анализ и его прил., 1997, том 31, выпуск 3, 35-43

DOI: https://doi.org/10.4213/faa473

Использование Общероссийского математического портала MathNet.Ru подразумевает, что вы прочитали и согласны с пользовательским соглашением

http://www . mathnet.ru/rus/agreement

Параметры загрузки:

IP : 3.80 .253 .173

26 апреля 2023 г., 12:39:47

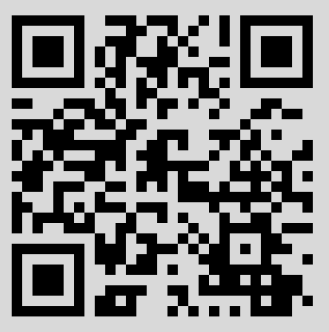


Функииональный анализ и его приложения

1997, т. 31, вып. 3, с. 35-43

УДК 517.98

\title{
Разделение серий для гиперболоидов
}

\author{
(c) 1997. В. Ф. МолЧАнов
}

В [2] была предложена некоторая программа для построения гармонического анализа на однородных многообразиях $G / H$ (так называемая программа Гельфанда-Гиндикина). Первый этап этой программы состоит в разложении пространства $L^{2}(G / H)$ на подпространства $L_{j}$, в каждом из которых действуют неприводимые унитарные представления одной серии. Следующие шаги должны дать естественное описание функций из $L_{j}$ (например, как граничных значений функций, голоморфных на некоторых комплексных многообразиях) и т. д.

В настоящей статье мы решаем задачу о разделении серий для вещественных гиперболоидов $X=S O_{0}(p, q) / S O_{0}(p, q-1)$. Как известно (см. $[4,7,14,15]$ и др.), в разложении квазирегулярного представления на гиперболоиде участвуют неприводимые унитарные представления двух серий: непрерывной и дискретной. Поэтому достаточно указать проектор на подпространство в $L^{2}(X)$, в котором действует одна из этих серий. Мы даем явные формулы для проектора на подпространство $L_{d}^{2}(X)$, в котором действует дискретная серия. В случае $q=2$ гиперболоид $X$ является границей Шилова некоторых комплексных многообразий, а дискретная серия распадается на две серии: аналитическую и антианалитическую. В работе [12] мы получили проекторы на каждую из этих серий, а также соответствующие ядра Коши-Сеге. Для полноты мы приводим в настоящей работе формулы также и для этих проекторов.

Заметим, что и в общем случае ( $q$ произвольно) дискретная серия связана $\mathrm{c}$ некоторой аналитичностью, см. формулы (1.1) и (3.4), (3.3) для проектора и для сферических функций.

Задача о разложении серий для гиперболоидов рассматривалась в работе С. Гиндикина $[10]$, в ней получен — в другой форме - проектор на дискретную серию в случае нечетного $n(n=p+q)$. Как видно из настоящей статьи, см. также [12], в этом круге вопросов случай нечетного $n$ проще, чем случай четного $n$; метод из [10] годится только для нечетного $n$.

\section{§1. Разделение серий для гиперболоидов}

Пусть в $\mathbb{R}^{n}$ задана билинейная форма

$$
[x, y]=-x_{1} y_{1}-\cdots-x_{p} y_{p}+x_{p+1} y_{p+1}+\cdots+x_{n} y_{n}
$$

* Работа выполнена при частичной поддержке Российского фонда фундаментальных исследований, грант 94-01-01603-а, а также Международного научного фонда и Правительства России, грант JC7100. Часть этой работы выполнена автором во время пребывания в Институте Миттаг-Леффлера (Дьюрсхольм, Швеция, январь-февраль 1996 г.) 
где $x=\left(x_{1}, \ldots, x_{n}\right), y=\left(y_{1}, \ldots, y_{n}\right)$ - векторы из $\mathbb{R}^{n}, n=p+q, p \geqslant 1$, $q \geqslant 2$. Гиперболоид $X$ в $\mathbb{R}^{n}$, определяемый уравнением

$$
[x, x]=1,
$$

является однородным пространством $G / H$, где $G=S O_{0}(p, q)$ - связная компонента единицы в группе линейных преобразований пространства $\mathbb{R}^{n}$, сохраняюших форму $[x, y]$, а $H=S O_{0}(p, q-1)$ - стационарная подгруппа точки

$$
x^{0}=(0, \ldots, 0,1) .
$$

Пусть $L^{2}(X)$ обозначает пространство $L^{2}$ на $X$ относительно $G$-инвариантной меры $d x=\left|x_{1}\right|^{-1} d x_{2} \ldots d x_{n}$. Пусть $U$ - представление группы $G$ в $L^{2}(X)$ сдвигами (квазирегулярное представление):

$$
(U(g) f)(x)=f(x g)
$$

(мы будем считать, что $G$ действует на $X$ справа).

Как мы уже говорили выше, представление $U$ разлагается по неприводимым унитарным представлениям непрерывной и (относительной) дискретной серий. Эта дискретная серия есть в точности первая дискретная серия представлений группы $G$, связанных с конусом, см. [5]. Пусть $L_{d}^{2}(X)$ обозначает подпространство в $L^{2}(X)$, в котором действует дискретная серия.

Для $q=2$ гиперболоид $X$ имеет структуру симметрического пространства эрмитова типа, см. $[11,13]$. В этом случае дискретная серия распадается на две серии: аналитическую и антианалитическую. Соответственно $L_{d}^{2}(X)$ распадается на два подпространства $L_{d,+}^{2}(X)$ и $L_{d,-}^{2}(X)$.

Пусть $E_{d}$ - проектор в $L^{2}(X)$ на $L_{d}^{2}(X)$. Для $q=2$ пусть, кроме того, $E_{d}^{ \pm}$ - проектор на $L_{d, \pm}^{2}(X)$, так что $E_{d}=E_{d}^{+}+E_{d}^{-}$. На пространстве Шварца $\mathscr{D}(X)$ бесконечно дифференцируемых функций с компактным носителем оператор $E_{d}$ задается с помощью ядра $E(x, y)$. Оно представляет собой обобщенную функцию на $X \times X$, инвариантную относительно диагонального действия группы $G$. Поэтому она вполне определяется функцией $E\left(x^{0}, x\right)$. Последняя есть обобщенная функция $E$ на $X$, инвариантная относительно $H$. Аналогично определяются обобщенные функции $E^{ \pm}$в случае $q=2$.

Основной результат настоящей статьи заключается в теоремах $1.1,1.2$, которые дают явные формулы для $E, E^{ \pm}$. Мы используем обобщенные функции на прямой из [3]: $x_{ \pm}^{\lambda},(x \pm i 0)^{\lambda}, \delta(x)$ (дельта-функция).

Теорема 1.1. Обобщенная функиия $E$ есть

$$
\sum_{ \pm} e^{ \pm i \pi(q-2) / 2} L\left(x_{n} \pm i 0\right)
$$

где для нечетного $n$

$$
\begin{gathered}
L(z)=c_{n}(z-1)^{-(n-1) / 2}, \\
c_{n}=(2 \pi)^{(-n-1) / 2} \Gamma((n-1) / 2),
\end{gathered}
$$

а для четного $n$

$$
\begin{gathered}
L(z)=c_{n}(z-1)^{-(n-1) / 2}(z+1)^{-(n-3) / 2} A(z), \quad z \in \mathbb{C} \backslash[-1,1], \\
c_{n}=\frac{1}{2}(2 \pi)^{-n / 2} \Gamma(n / 2) ;
\end{gathered}
$$


здесь $A(z)=A_{(n-4) / 2}(z), a A_{m}(z)$ - следуюший многочлен степени $m$ om $z$ :

$$
A_{m}(z)=\sum_{k=0}^{m} \frac{1}{2^{k}}\left(\begin{array}{c}
m \\
k
\end{array}\right)\left(\begin{array}{c}
k \\
{[k / 2]}
\end{array}\right) z^{m-k}
$$

[a] есть иелая часть числа $а$.

ТеоремА 1.2. Для $q=2$ обобшенные функиии $E^{ \pm}$имеют следуюший вид:

$$
E^{ \pm}(x)=L\left(x_{n} \pm i 0 \cdot x_{n-1}\right)=\lim _{\varepsilon \rightarrow+0} L\left(x_{n} \pm i \varepsilon x_{n-1}\right)
$$

где $L(z)$ имеет тот же вид, что и выъше.

Положим для краткости

$$
\nu=(n-3) / 2 .
$$

Как следует из [3], явные выражения для $E, E^{ \pm}$можно записать в несколько иной форме. А именно, $E$ есть аналитическое продолжение по $\mu$ в точку $\mu=\nu$ следующей обобщенной функщии, зависящей от комплексного параметра $\mu$ :

$$
2 c_{n}\left\{\cos \frac{q-2}{2} \pi \cdot\left(x_{n}-1\right)_{+}^{-\mu-1}+\cos \left(\frac{q}{2}-\mu\right) \pi \cdot\left(x_{n}-1\right)_{-}^{-\mu-1}\right\},
$$

если $n$ нечетно, и

$$
\begin{aligned}
& 2 c_{n}\left\{\cos \frac{q-2}{2} \pi \cdot(\right.\left.x_{n}-1\right)_{+}^{-\mu-1}\left(x_{n}+1\right)_{+}^{-\mu}+\cos \left(\frac{q}{2}-\mu\right) \pi \cdot\left(x_{n}-1\right)_{-}^{-\mu-1}\left(x_{n}+1\right)_{+}^{-\mu} \\
&\left.+\cos \left(\frac{q}{2}-2 \mu\right) \pi \cdot\left(x_{n}-1\right)_{-}^{-\mu-1}\left(x_{n}+1\right)_{-}^{-\mu}\right\} A\left(x_{n}\right), \quad(1.10)
\end{aligned}
$$

если $n$ четно. Это позволяет, в частности, найти носитель обобщенной функции $E$ :

\begin{tabular}{|c|c|}
\hline$(p, q) \bmod 2$ & $\operatorname{supp} E$ \\
\hline \hline$(0,1)$ & $x_{n}=1$ \\
\hline$(1,0)$ & $X$ \\
\hline$(0,0)$ & $\left|x_{n}\right| \geqslant 1$ \\
\hline$(1,1)$ & $\left|x_{n}\right| \leqslant 1$ \\
\hline
\end{tabular}

Мы видим, что в случае $(p, q) \equiv(0,1)$ (здесь и дальше знак сравнения означает сравнение по модулю 2) обобщенная функция $E$ сосредоточена на многообразии меньшей размерности - на конусе $x_{n}=1$ (он состоит из особых орбит подгруппы $H$ на $X$ ).

Для $q=2$ обобщенные функции $E^{ \pm}$суть аналитическое продолжение по $\mu$ в точку $\mu=\nu$ следующих обобщенных функций, зависящих от комплексного параметра $\mu$ :

$$
\begin{aligned}
& c_{n}\left\{\left(x_{n}-1\right)_{+}^{-\mu-1}-\cos \pi \mu \cdot\left(x_{n}-1\right)_{-}^{-\mu-1} Y\left(-x_{n}-1\right)\right. \\
&\left.-\left[e^{\mp i \pi \mu} Y\left(x_{n-1}\right)+e^{ \pm i \pi \mu} Y\left(-x_{n-1}\right)\right]\left(x_{n}-1\right)_{-}^{-\mu-1} Y\left(x_{n}+1\right)\right\},
\end{aligned}
$$


если $n$ нечетно, и

$$
\begin{aligned}
& c_{n}\left\{\left(x_{n}-1\right)_{+}^{-\mu-1}\left(x_{n}+1\right)_{+}^{-\mu}\right. \\
& \quad-\left[e^{\mp i \pi \mu} Y\left(x_{n-1}\right)+e^{ \pm i \pi \mu} Y\left(-x_{n-1}\right)\right]\left(x_{n}-1\right)_{-}^{-\mu-1}\left(x_{n}+1\right)_{+}^{-\mu} \\
& \left.\quad-\cos 2 \mu \pi\left(x_{n}-1\right)_{-}^{-\mu-1}\left(x_{n}+1\right)_{-}^{-\mu}\right\} A\left(x_{n}\right),
\end{aligned}
$$

если $n$ четно, где $Y(t)$ - функция Хевисайда $(Y(t)=1$ для $t>0, Y(t)=0$ для $t<0)$. Носитель обеих функций $E^{ \pm}$есть весь гиперболоид $X$. Отметим, что для $q=2$ сечение гиперболоида $X$ плоскостью $x_{n}=c$ для $|c|<1$ состоит из двух $H$-орбит: $\pm x_{n-1} \geqslant \sqrt{1-c^{2}}$. Это объясняет появление $x_{n-1}$ в формулах (1.7), (1.11), (1.12).

Для $q=2$ запишем еще разность проекторов:

$$
Q_{d}=E_{d}^{+}-E_{d}^{-}
$$

(это аналог преобразования Гильберта). Пусть $Q-$ соответствующая обобщенная функция. Тогда (см. [12]), если $n$ нечетно, то

$$
Q=\left.2 i c_{n} \sin \mu \pi \cdot\left(x_{n}-1\right)_{-}^{-\mu-1} Y\left(x_{n}+1\right)\left[Y\left(x_{n-1}\right)-Y\left(-x_{n-1}\right)\right]\right|_{\mu=\nu},
$$

а если $n$ четно, то

$$
Q=\left.2 i c_{n} \sin \mu \pi \cdot\left(x_{n}-1\right)_{-}^{-\mu-1}\left(x_{n}+1\right)_{+}^{-\mu} A\left(x_{n}\right)\left[Y\left(x_{n-1}\right)-Y\left(-x_{n-1}\right)\right]\right|_{\mu=\nu} .
$$

Носитель обобщенной функции $Q$ для нечетного $n$ есть множество $x_{n}=1$, а для четного $n$ - множество $\left|x_{n}\right| \leqslant 1$. Особенно приятно оператор $Q_{d}$ выглядит для $n=3(p=1, q=2)$ (см. [10]):

$$
\left(Q_{d} f\right)(x)=\frac{1}{2 \pi i}\left\{\int_{-\infty}^{\infty} f\left(x+t e_{x}^{-}\right) \frac{d t}{t}-\int_{-\infty}^{\infty} f\left(x+t e_{x}^{+}\right) \frac{d t}{t}\right\},
$$

где $e_{x}^{ \pm}$- направляющие векторы двух прямолинейных образующих гиперболоида $X$, проходящих через точку $x$, такие, что их первая координата равна 1 и векторы $x, e_{x}^{+}, e_{x}^{-}$образуют правую тройку. Таким образом, оператор $2 \pi i Q_{d}$ есть разность интегралов по прямолинейным образующим, проходящим через данную точку, взятых по мере, инвариантной относительно стационарной подгруппы этой точки.

Остальная часть статьи посвящена доказательству теоремы 1.1. Теорема 1.2, а также формулы $(1.13),(1.14)$ доказаны в работе [12].

\section{§2. Усреднение}

Усреднение $M$ сопоставляет каждой функции $f \in \mathscr{D}(X)$ функцию $(M f)(c)$ на $\mathbb{R}$, определенную следующим образом:

$$
(M f)(c)=\int_{X} f(x) \delta\left(x_{n}-c\right) d x .
$$

Это отображение сводится к усреднению функций в $\mathbb{R}^{n-1}$ по поверхностям уровня квадратичной формы сигнатуры $(p, q-1)$, см. $[3,16]$. Определим функ- 
цию $\theta(c)$ формулой

$$
\theta(c)= \begin{cases}(c-1)_{+}^{\nu}, & q \equiv 1, \\ (c-1)_{-}^{\nu}, & (p, q) \equiv(0,0), \\ (c-1)^{\nu} \ln |c-1|, & (p, q) \equiv(1,0) .\end{cases}
$$

Образ $\mathfrak{M}$ отображения $M$ состоит из функций $h(c)$ на $\mathbb{R}$, которые можно представить в виде (см. [9])

$$
h(c)=u(c)+\theta(c) v(c)+\theta(-c) w(c),
$$

где $u, v, w-$ функции из $\mathscr{D}(\mathbb{R})$, причем $\operatorname{supp} v \subset(-1, \infty), \operatorname{supp} w \subset(-\infty, 1)$. Можно так ввести топологию в $\mathfrak{M}$, что отображение $M: \mathscr{D}(X) \rightarrow \mathfrak{M}$ станет непрерывным, см., например, [16]. Сопряженное отображение $M^{\prime}$ есть линейный гомеоморфизм сопряженного пространства $\mathfrak{M}^{\prime}$ в пространство обобщенных функций на $X$, инвариантных относительно $H$ (для $p>1, q>2$ это есть отображение «на»).

Пусть $\mathfrak{M}_{+}, \mathfrak{M}_{-}$— подпространства в $\mathfrak{M}$, состоящие из функций $h$ с носителями в $(-1, \infty),(-\infty, 1)$ соответственно.

Мы можем записать значение обобщенной функции $E$ на $f$, т.е. значение $\left(E_{d} f\right)\left(x^{0}\right)$, с помощью $M f$. Для этого надо в функциях $(1.9),(1.10)$ взять $c$ вместо $x_{n}$, умножить их на $(M f)(c)$, проинтегрировать при $\operatorname{Re} \mu<1$ по всей оси $(c \in \mathbb{R})$ и продолжить в точку $\mu=\nu$.

Предъявим некоторые функционалы из $\mathfrak{M}^{\prime}$, которые нам потребуются для описания сферических функций в 33 .

Рассмотрим следующие функции комплексного переменного $z$, зависящие от двух комплексных параметров $\sigma, \mu$ и выражающиеся через функции Лежандра первого и второго рода (см. [1, гл. 3$])$ :

$$
\begin{aligned}
& \varphi_{\sigma \mu}(z)=\left(z^{2}-1\right)^{-\mu / 2} P_{\sigma+\mu}^{\mu}(z), \\
& \psi_{\sigma \mu}(z)=e^{-i \mu \pi}\left(z^{2}-1\right)^{-\mu / 2} Q_{\sigma+\mu}^{\mu}(z),
\end{aligned}
$$

где $z \in \mathbb{C} \backslash(-\infty, 1]$. На разрезе $(-\infty, 1]$ определим эти функции как полусумму предельных значений сверху и снизу. Эти функции удовлетворяют дифференциальному уравнению

$$
\left(z^{2}-1\right) \frac{d^{2} y}{d z^{2}}+(2 \mu+2) z \frac{d y}{d z}=\sigma(\sigma+2 \mu+1) y .
$$

Отметим, что при $\mu=\nu$ это уравнение с заменой $z$ на $c$ превращается в уравнение для прообразов в $\mathfrak{M}^{\prime}$ сферических функций на $X$.

Особые точки $c= \pm 1$ уравнения $(2.2)$ разбивают $\mathbb{R}$ на три интервала: $I_{1}=$ $(1, \infty), I_{2}=(-1,1), I_{3}=(-\infty,-1)$.

Для функции $f(c)$ на $\mathbb{R}$ положим $\hat{f}(c)=f(-c)$.

Нам потребуются некоторые соотношения на $I_{2}$, они выводятся из $[1,3.4(14)$, (15)]:

$$
\begin{aligned}
& \varphi_{\sigma \mu}(c)=\cos (\sigma+2 \mu) \pi \cdot \widehat{\varphi}_{\sigma \mu}(c)-\frac{2}{\pi} \sin (\sigma+2 \mu) \pi \cdot \widehat{\psi}_{\sigma \mu}(c) \\
& \psi_{\sigma \mu}(c)=-\frac{\pi}{2} \sin (\sigma+2 \mu) \pi \cdot \widehat{\varphi}_{\sigma \mu}(c)-\cos (\sigma+2 \mu) \pi \cdot \widehat{\psi}_{\sigma \mu}(c)
\end{aligned}
$$


Пусть $F$ - одна из функций $\varphi_{\sigma \mu}, \psi_{\sigma \mu}, \widehat{\varphi}_{\sigma \mu}, \widehat{\psi}_{\sigma \mu}$. Для $j=1,2,3$ определим функционал $F^{(j)}$ из $\mathfrak{M}^{\prime}$ с помощью интеграла:

$$
\left(F^{(j)}, h\right)=\int_{I_{j}} F(c) h(c) d c, \quad h \in \mathfrak{M} .
$$

Интеграл абсолютно сходится при $\operatorname{Re} \mu<1$, а на остальные $\mu$ функционал $F^{(j)}$ продолжается по аналитичности до мероморфной функции - с возможными полюсами в точках $\mu=1,2, \ldots$ и $\mu=\nu+1, \nu+2, \ldots$. Сумму $F^{(i)}+F^{(j)}$ будем обозначать $F^{(i j)}$. Нас специально интересует значение $\mu=\nu$. Если $\nu$ не целое (т.е. $n$ четно), то все эти функционалы регулярны (не имеют особенности) в точке $\mu=\nu$. Если $\nu$ целое (т.е. $n$ нечетно), то $\varphi_{\sigma \mu}^{(2)}, \widehat{\varphi}_{\sigma \mu}^{(2)}$ регулярны в точке $\mu=\nu$, а $\psi_{\sigma \mu}^{(j)}, \widehat{\psi}_{\sigma \mu}^{(j)}$ - нет, но суммы $\psi_{\sigma \mu}^{(12)}$ и $\widehat{\psi}_{\sigma \mu}^{(23)}$ регулярны в точке $\mu=\nu$ на подпространствах $\mathfrak{M}_{+}$и $\mathfrak{M}_{-}$соответственно.

Определим еще функционалы $\psi_{\sigma \mu}^{ \pm}$из $\mathfrak{M}^{\prime}$ с помощью предельного перехода:

$$
\left(\psi_{\sigma \mu}^{ \pm}, h\right)=\lim _{\varepsilon \rightarrow+0} \int_{-\infty}^{\infty} \psi_{\sigma \mu}(c \pm i \varepsilon) h(c) d c .
$$

С помошью $[1,3.4(8), 3.3(12)]$ мы можем выразить предельные значения $\psi_{\sigma \mu}(c \pm$ $i 0)$ через $\psi_{\sigma \mu}(c), \ldots$ Отсюда мы получаем выражения функционалов $\psi_{\sigma \mu}^{ \pm}$через функционалы $\psi_{\sigma \mu}^{(j)}, \ldots$ :

$$
\begin{aligned}
\psi_{\sigma \mu}^{ \pm} & =\psi_{\sigma \mu}^{(12)}+e^{\mp i \pi(\sigma+2 \mu+1)} \widehat{\psi}_{\sigma \mu}^{(3)} \mp \frac{i \pi}{2} \varphi_{\sigma \mu}^{(2)} \\
& =\psi_{\sigma \mu}^{(1)}+e^{\mp i \pi(\sigma+2 \mu+1)} \widehat{\psi}_{\sigma \mu}^{(23)} \mp \frac{i \pi}{2} \widehat{\varphi}_{\sigma \mu}^{(2)}
\end{aligned}
$$

(выражение (2.7) получается из (2.6) с помощью (2.3), (2.4)). Отсюда видно, что функционалы $\psi_{\sigma \mu}^{ \pm}$не имеют особенности при $\mu=\nu,-$ в самом деле, как было сказано в предыдущем абзаце, функционалы в правых частях $(2.6),(2.7)$ не имеют особенности при $\mu=\nu$ на $\mathfrak{M}_{+}, \mathfrak{M}_{-}$соответственно.

\section{§3. Сферические функции дискретной серии}

Один из вариантов формулы Планшереля для гиперболоида $X$ состоит в том, что дельта-функция $\delta$ на $X$, сосредоточенная в точке $x^{0}$, разлагается (в смысле обобщенных функций) по сферическим функциям, отвечающим неприводимым унитарным представлениям группы $G$. Сферические функции на $X$ были определены и вычислены в $[6]$; см. также $[7,9]$ (это обобщенные функции на $X$, инвариантные относительно $H$ и собственные для оператора Лапласа-Бельтрами). Если в упомянутой формуле мы соберем вместе слагаемые, отвечающие представлениям дискретной серии, мы как раз получим разложение обобщенной функции $E$ из $\S 1$. Согласно, например, [7] и $[9, \S 30]$, эта дискретная часть формулы Планшереля имеет вид

$$
E=a \sum_{r=-[\nu]}^{\infty}(2 r+n-2) E_{r}
$$


где суммирование происходит по целым $r>(2-n) / 2$,

$$
a=2(2 \pi)^{(-n-1) / 2},
$$

а обобщенные функции $E_{r}$ (сферические функции дискретной серии) явно указаны. В теореме 3.1 будет указан удобный для нас здесь явный вид функций $E_{r}$. (При $q=2$ обобщенные функции $E_{r}$ распадаются в сумму $E_{r}^{+}+E_{r}^{-}$сферических функций, отвечающих представлениям аналитической и антианалитической серий.)

Рассмотрим следующие функционалы из $\mathfrak{M}^{\prime}$ :

$$
V_{r}=\frac{1}{2} \sum_{ \pm} e^{ \pm i \pi(q-2) / 2} \psi_{r \nu}^{ \pm}
$$

ТЕорема 3.1. Сферические функчии $E_{r}$ дискретной серии $(r \in \mathbb{Z}, r>$ $(2-n) / 2)$ вьражаются следуюшим образом:

$$
E_{r}=M^{\prime} V_{r}
$$

Для $r \geqslant 0$ теорема доказана в [7]; для $r<0$ она доказывается аналогично.

Напишем подробные выражения для $E_{r}$ через $\psi_{r \nu}^{(j)}, \ldots$ Для этого подставим в (3.3) выражения (2.6), (2.7) с $\mu=\nu$ и получим

$$
\begin{aligned}
E_{r} & =M^{\prime}\left\{\cos \frac{q-2}{2} \pi\left[\psi_{r \nu}^{(12)}+(-1)^{r+n} \widehat{\psi}_{r \nu}^{(3)}\right]+\frac{\pi}{2} \sin \frac{q-2}{2} \pi \cdot \varphi_{r \nu}^{(2)}\right\} \\
& =M^{\prime}\left\{\cos \frac{q-2}{2} \pi\left[\psi_{r \nu}^{(1)}+(-1)^{r+n} \widehat{\psi}_{r \nu}^{(23)}\right]+\frac{\pi}{2} \sin \frac{q-2}{2} \pi \cdot \widehat{\varphi}_{r \nu}^{(2)}\right\} .
\end{aligned}
$$

Выражения (3.5), (3.6) удобны на $\mathfrak{M}_{+}, \mathfrak{M}_{-}$соответственно.

\section{§4. Доказательство теоремы 1.1}

Начнем с формулы 3.8(3) из [1], которая в наших обозначениях принимает вид

$$
(2 \sigma+2 \mu+1) \psi_{\sigma \mu}(z)=\psi_{\sigma-2, \mu+1}(z)-\psi_{\sigma, \mu+1}(z) .
$$

Положим здесь $\sigma=r \in \mathbb{Z}, \mu=\nu$, см. (1.8), и просуммируем по $-[\nu] \leqslant r \leqslant N$ :

$$
\sum_{r=-[\nu]}^{N}(2 r+n-2) \psi_{r \nu}(z)=\Psi(z)-\psi_{N-1, \nu+1}(z)-\psi_{N, \nu+1}(z),
$$

где

$$
\begin{array}{ll}
\Psi(z)=\psi_{-\nu-2, \nu+1}(z)+\psi_{-\nu-1, \nu+1}(z), & n \text { нечетно, } \\
\Psi(z)=\psi_{-\nu-3 / 2, \nu+1}(z)+\psi_{-\nu-1 / 2, \nu+1}(z), & n \text { четно. }
\end{array}
$$

Предельные значения этой функции $\Psi(z)$ на $\mathbb{R}$ сверху и снизу дают два функционала $\Psi^{ \pm}$из $\mathfrak{M}^{\prime}$, cp. $(2.5)$. Положив $z=c \pm i 0$ в $(4.1)$, умножив на $e^{ \pm i \pi(q-2) / 2}$ и просуммировав по \pm , мы получим в силу (3.3) равенство функционалов:

$$
\sum_{r=-[\nu]}^{N}(2 r+n-2) V_{r}=W-V_{N-1}-V_{N}
$$


где

$$
W=\frac{1}{2} \sum_{ \pm} e^{ \pm i \pi(q-2) / 2} \Psi^{ \pm}
$$

В [7] доказано, что $V_{k} \rightarrow 0$ при $k \rightarrow \infty$. Поэтому, переходя к пределу в (4.4) при $N \rightarrow \infty$, мы получим

$$
\sum_{r=-[\nu]}^{\infty}(2 r+n-2) V_{r}=W .
$$

Сопоставляя (4.6), (3.1), (3.4), находим

$$
E=a M^{\prime} W,
$$

где $a$ дается формулой (3.2). Таким образом, в силу (4.5) остается вычислить $\Psi(z)$.

Пусть сначала $n$ нечетно ( $\nu$ целое). Запишем функции $\psi$ из $(4.2)$ через функции Лежандра (см. (2.1)):

$$
\begin{aligned}
& \psi_{-\nu-2, \nu+1}(z)=e^{-i(\nu+1) \pi}\left(z^{2}-1\right)^{-(\nu+1) / 2} Q_{-1}^{\nu+1}(z), \\
& \psi_{-\nu-1, \nu+1}(z)=e^{-i(\nu+1) \pi}\left(z^{2}-1\right)^{-(\nu+1) / 2} Q_{0}^{\nu+1}(z) .
\end{aligned}
$$

Эти функции Лежандра мы вычисляем с помощью формул $3.2(35),(32)$ из [1] соответственно, после чего по (4.2) мы получаем

$$
\Psi(z)=\Gamma(\nu+1)(z-1)^{\nu+1} .
$$

Стало быть, по (4.5)

$$
W=\frac{1}{2} \Gamma(\nu+1) \sum_{ \pm} e^{ \pm i \pi(q-2) / 2}(c-1 \pm i 0)^{-\nu-1} .
$$

Вместе с (4.7) это дает формулы (1.1)-(1.3).

Пусть теперь $n$ четно ( $\nu$ полуцелое). Запишем функции $\psi$ из (4.3) через функции Лежандра:

$$
\begin{aligned}
& \psi_{-\nu-3 / 2, \nu+1}(z)=e^{-i(\nu+1) \pi}\left(z^{2}-1\right)^{-(\nu+1) / 2} Q_{-1 / 2}^{\nu+1}(z), \\
& \psi_{-\nu-1 / 2, \nu+1}(z)=e^{-i(\nu+1) \pi}\left(z^{2}-1\right)^{-(\nu+1) / 2} Q_{1 / 2}^{\nu+1}(z) .
\end{aligned}
$$

Применив к первой из них формулу 3.8(7) из [1], а ко второй — формулу 3.8(8) из [1], мы получим

$$
\Psi(z)=e^{-i \nu \pi}\left(z^{2}-1\right)^{-(\nu+2) / 2}(z+1)\left\{(\nu-1 / 2) Q_{1 / 2}^{\nu}(z)+(\nu+1 / 2) Q_{-1 / 2}^{\nu}(z)\right\} .
$$

Положим для краткости $m=(n-4) / 2$, это целое число. Выразив обе функции Лежандра в (4.8) через гипергеометрическую функцию по формуле $3.2(8)$ из [1], получим

$$
\begin{aligned}
\Psi(z)=\sqrt{\frac{\pi}{2}} \Gamma\left(\frac{n}{2}\right)\left(z^{2}-1\right)^{-\nu-1}(z+1) & \left\{\frac{m}{2} z^{m-1} F\left(\frac{-m+1}{2}, \frac{-m+2}{2} ; 2 ; z^{-2}\right)\right. \\
& \left.+z^{m} F\left(\frac{-m}{2}, \frac{-m+1}{2} ; 1 ; z^{-2}\right)\right\} .
\end{aligned}
$$


Функция, стоящая в фигурных скобках в (4.9), есть многочлен от z степени $m$ : гипергеометрические ряды здесь обрываются. Выписывая их коэффициенты, мы убеждаемся в том, что упомянутый многочлен есть в точности $A_{m}(z)$, см. $(1.6)$, так что

Стало быть,

$$
\Psi(z)=\sqrt{\pi / 2} \Gamma(n / 2)(z-1)^{-\nu-1}(z+1)^{-\nu} A_{m}(z)
$$

$$
W=\frac{1}{2} \sqrt{\frac{\pi}{2}} \Gamma\left(\frac{n}{2}\right) \sum_{ \pm} e^{ \pm i \pi(q-2) / 2}(c-1 \pm i 0)^{-\nu-1}(c+1 \pm i 0)^{-\nu} A_{m}(c) .
$$

Вместе с (4.7) это дает (1.1), (1.4), (1.5).

\section{ЛИТЕРАТУРА}

1. Бейтмен Г., Эрдейи A. Высшие трансцендентные функции (гипергеометрическая функция, функции Лежандра). Наука, М. (1965).

2. Гельфанд И. М., Гиндикин С. Г. Комплексные многообразия, остовы которых - полупростые вещественные группы Ли, и аналитические дискретные серии представлений. Функц. анализ и его прил., 11, вып. 4, $19-27$ (1977).

3. Гельфанд И. М., Шилов Г. Е. Обобщенные функции и действия над ними. Физматгиз, М. (1958).

4. Молчанов В. Ф. Аналог формулы Планшереля для гиперболоидов. ДАН СССР, 183, №2, 288-291 (1968).

5. Молчанов В. Ф. Представления псевдоортогональной группы, связанные с конусом. Матем. сб., 81 (123), №3, 358-375 (1970).

6. Молчанов В. Ф. Сферические функции на гиперболоидах. Матем. сб., 99 (141), №2, 139-161 (1976).

7. Молчанов В. Ф. Формула Планшереля для гиперболоидов. Труды Матем. инта им. В. А. Стеклова, 147, 65-85 (1980).

8. Молчанов В. Ф. Квантование на мнимой плоскости Лобачевского. Функц. анализ и его прил., 14, №2, 73-74 (1980).

9. Молчанов $B . \Phi$. Гармонический анализ на однородных пространствах. В кн.: Итоги науки и техники, Современные проблемы математики, т. 59, ВИНИТИ, M. (1990), c. 5-144.

10. Gindikin S. Conformal analysis on hyperboloids. J. Geom. Phys., 10, 175-184 (1993).

11. Matsumoto $S$. Discrete series for an affine symmetric space. Hiroshima Math. J., 11, 53-79 (1981).

12. Molchanov $V$. F. Holomorphic discrete series for hyperboloids of Hermitian type. Univ. Leiden Preprint W 94-13 (1994), готовится к печати в J. Funct. Anal., 145 (1977).

13. Olafsson G., Ørsted B. The holomorphic discrete series for affine symmetric spaces, I. J. Funct. Anal., 81, No. 1, 126-159 (1988).

14. Rossmann $W$. Analysis on real hyperbolic spaces. J. Funct. Anal., 30, No. 3, 448-477 (1978).

15. Strichartz R. S. Harmonic analysis on hyperboloids. J. Funct. Anal., 12, No. 4, $341-383$ (1973).

16. Tengstrand $A$. Distributions invariant under an orthogonal group of arbitrary signature. Math. Scand., 8, 201-218 (1960). 